\title{
Cyclic Boosting - an explainable supervised machine learning algorithm
}

\author{
Felix Wick ${ }^{* 1}$, Ulrich Kerzel ${ }^{\dagger 2}$, and Michael Feindt ${ }^{\ddagger 1}$ \\ $1_{\text {Blue Yonder GmbH (Karlsruhe, Germany) }}$ \\ ${ }^{2}$ IUBH Internationale Hochschule (Erfurt, Germany)
}

Supervised machine learning algorithms have seen spectacular advances and surpassed human level performance in a wide range of specific applications. However, using complex ensemble or deep learning algorithms typically results in black box models, where the path leading to Nindividual predictions cannot be followed in detail. In -order to address this issue, we propose the novel "Cyclic ZBBoosting" machine learning algorithm, which allows to efficiently perform accurate regression and classification tasks while at the same time allowing a detailed understanding of how each individual prediction was made.

Keywords: machine learning, explainable algorithms, demand forecasting

DOI: 10.1109/ICMLA.2019.00067

e?

\section{Introduction}

In many practical applications one is concerned with prendicting a quantity $Y$ (target or label) from a set of features $\sim \boldsymbol{X}$, i.e. one needs to estimate the conditional $p(Y \mid \boldsymbol{X})$ of $\checkmark$ the joint probability density distribution $p(Y, \boldsymbol{X})$ when The values $\vec{x}$ of the feature variables $\boldsymbol{X}$ are observed. In supervised machine learning, one uses e.g. neural networks like NeuroBayes [1] to build a model for the conditional, i.e. $\tilde{p}(Y \mid \boldsymbol{X}, \vec{\theta})$, where $\vec{\theta}$ is a set of parameters learned from the training data and represents all observed knowledge of the joint probability distribution $p(Y, \boldsymbol{X})$.

-7 Although the predictions obtained from the machine learning model are in general very accurate, the exact path how an individual prediction was calculated is typically not observable in complex ensemble or deep learning models. Being able to explain how individual predictions and decisions were made can be a mandatory legal requirement in many sectors such as finance and insurance and is highly desirable in others such as medicine or retail to build trust in the machine learning model.

In addition, most machine learning algorithms struggle to learn rare events, as they are not representative of the bulk of the data and are often over-regularized, even though in practical applications these effects may play a major role.

\footnotetext{
*felix.wick@blueyonder.com

$\dagger$ u.kerzel@iubh-fernstudium.de

${ }^{\ddagger}$ michael.feindt@blueyonder.com
}

In order to address both the explainability and handling of rare effects, a novel machine learning algorithm called "Cyclic Boosting" is proposed, which is able to learn a model $\tilde{p}(Y \mid \boldsymbol{X}, \vec{\theta})$ efficiently and accurately, while allowing to precisely follow the path how individual predictions were made.

As will become apparent below, Cyclic Boosting can be categorized as a generalized additive model [2, where the target $Y$ belongs to the family of exponential distributions such as the Poisson, Gaussian, or Bernoulli distribution, together with a suitable link function. As such, the Cyclic Boosting algorithm can be used in the following three scenarios:

- Multiplicative regression mode: $Y \in[0, \infty)$

- Additive regression mode: $Y \in(-\infty, \infty)$

- Classification mode: $Y \in[0,1]$

In the following, the multiplicative regression mode is described in detail first and for the other two modes only modifications are highlighted afterwards.

\section{Literature Review}

Reaching human-level performance using machine learning techniques in specific applications has also increased the interest in interpretability of algorithm-driven decisions in recent years. However, due to the opaque nature of most of the complex models it remains largely unexplained how these decisions are reached.

Several approaches exist to address this situation, e.g. Molnar 3] gives a good overview. In general, one can either use a complex black box model and subsequently apply model-agnostic interpretation tools or build an explainable model.

For black box models, the importance of individual input features can be determined using e.g. Shapley's gametheoretic approach [4, 5] or permutation importance [6,7]. Google's What-If 8 allows to probe the behavior of a machine learning model if certain aspects or inputs are changed. Partial dependency plots [9] can be used to visualize the relationship between the target and selected features and illustrate if e.g. the dependency is non-linear or 
monotonous. Multiple visualization techniques have been developed in particular to understand the way deep neural networks process images, such as partial occlusion [10] or saliency maps 11]. These approaches are undoubtedly invaluable tools to understand the inner workings of black box models as well as the correlation between input features and predictions. However, they cannot address the black box character on a fundamental level and therefore don't allow for fully explainable models.

Surrogate models can be used to build explainable models out of black box models. For instance, LIME [12] uses linear models to derive an explainable model which is faithful locally around each prediction. While these predictions are locally explainable, the model itself remains a black box model.

The simplest fully explainable model is the linear or logistic regression where the target is represented by a sum of linear features and a Gaussian noise term $\epsilon$ : $y=$ $\sum_{i} \alpha_{i} x_{i}+\epsilon$. Once all coefficients $\alpha_{i}$ are determined from data, each prediction can be evaluated in terms of the features and their coefficients. However, this approach suffers from many short-comings, e.g. all features are assumed to be linear with constant variance, as well as independent from each other. Consequently, this simple approach is rarely sufficient in practical applications.

General Linear Models (GLM) 13 extend this approach and are useful for a wider range of models. However, they still retain their linear character. More generally, General Additive Models (GAM) [2] replace the linear term with a function $f_{i}\left(x_{i}\right)$ which allows for the modeling of non-linear effects. In general, GAMs are described by $g(E[y])=\beta_{0}+\sum f_{j}\left(x_{j}\right)$, where $g$ is called the link function and $f_{j}$ is some function which operates on the features $x_{j}$. In case of GLMs, $f$ is constrained to be linear. While GAMs are not as easily interpretable as a simple linear regression, they retain most of the benefits while allowing to model complex relationships found in concrete application scenarios. Recently, Microsoft released Interprete 14, which includes a GAM with pairwise interactions for each feature variable [15], i.e. $g(E[y])=\beta_{0}+\sum_{j} f_{j}\left(x_{j}\right)+\sum_{i \neq j} f_{i j}\left(x_{i}, x_{j}\right)$.

Considering other supervised learning approaches, single decision trees $[6$ are fully explainable, but are often not sufficiently performant in practical applications. While amending them to ensemble methods by means of e.g. bagging or boosting techniques can improve the performance of tree-based methods significantly, it greatly reduces the explainability of the models as well. The same holds for support vector machines (SVM) [16]: For a linear kernel, the SVM weights define the hyperplane which separates two classes. In case of low dimensional feature space, this can be used to gain insights into the relative importance of the input variables. However, in case of high-dimensional spaces this becomes more difficult and more general nonlinear kernels do not allow for easy interpretation of the SVM decision boundaries. Artificial neural networks are typically considered as black box models due to their nonlinear transfer function modifying the output of individual neurons.

\section{The Cyclic Boosting algorithm}

\subsection{General approach}

The main idea behind Cyclic Boosting is that each individual feature $X_{j}$ from $\boldsymbol{X}=\left(X_{1}, X_{2}, \ldots, X_{p}\right)$ contributes in a specific way to the prediction of the target $\hat{Y}$. If all contributions can be calculated on a granular level, each prediction $\hat{y}_{i}$ for a given observation $i$ can be transparently interpreted by analyzing how much each feature $X_{j}$ for the observed values $x_{j, i}$ contributes to the prediction.

To achieve the required granularity, each feature $X_{j}$ is first binned appropriately: Categorical features retain their original categories, whereas continuous features are discretized such that each bin has the same width (equidistant binning) or contains the approximately same number of observations. In the following, bins are denoted by $b_{j}^{k}$, i.e. bin $k=1, \ldots, n$ for feature $X_{j}$. During the training of the supervised machine learning model, each feature, containing its various bins, is considered in turn and an appropriate modification to the prediction $\hat{Y}$ of the target $Y$ is calculated. This process is repeated iteratively until a stopping criterion is met, e.g. the maximum number of iterations or no further improvement of an error metric such as the mean absolute deviation (MAD) or mean squared error (MSE).

\subsection{Multiplicative regression mode}

In the multiplicative regression mode of Cyclic Boosting, the target variable is in the range $Y \in[0, \infty)$. The predicted values of the target variable, denoted by $\hat{y}_{i}$, are calculated from given observations $\vec{x}_{i}$ of a set of feature variables $\boldsymbol{X}$ in the following way.

$$
\hat{y}_{i}=\mu \cdot \prod_{j=1}^{p} f_{j}^{k} \quad \text { with } k=\left\{x_{j, i} \in b_{j}^{k}\right\}
$$

Here, $f_{j}^{k}$ are the model parameters for each feature $j$ and bin $k$. For any concrete observation $i$, the index $k$ of the bin is determined by the observation of $x_{j, i}$ and the subsequent look-up into which bin this observation falls. The global average $\mu$ is calculated from all observed target values $y$ taken across the entire training data.

If one assumes that the target variable $Y$ is generated as the mean of a Poisson (or more general, negative binomial) distribution and the logarithm $l n$ is used as the link function, eqn. 1 can be inferred from the structure of a generalized additive model by applying the inverse link function.

The model parameters $f_{j}^{k}$ are determined from the training data according to the following meta-algorithm:

1. Calculate the global average $\mu$ from all observed $y$ across all bins $k$ and features $j$.

2. Initialize the factors $f_{j}^{k} \leftarrow 1$

3. Cyclically iterate through features $j=1, \ldots, p$ and calculate in turn for each bin $k$ the partial factors $g$ and corresponding aggregated factors $f$, where indices 
$t$ (current iteration) and $\tau$ (current or preceding iteration) refer to iterations of full feature cycles as the training of the algorithm progresses:

$$
g_{j, t}^{k}=\frac{\sum_{x_{j, i} \in b_{j}^{k}} y_{i}}{\sum_{x_{j, i} \in b_{j}^{k}} \hat{y}_{i, \tau}} \text { where } f_{j, t}^{k}=\prod_{s=1}^{t} g_{j, s}^{k}
$$

This means $g$ is the factor that is multiplied to $f_{t-1}$ in each iteration. Here, $\hat{y}_{\tau}$ is calculated according to eqn. 1 with the current values of the aggregated factors $f$ :

$$
\hat{y}_{i, \tau}=\mu \cdot \prod_{j=1}^{p} f_{j, \tau}^{k}
$$

To be precise, the determination of $g_{j, t}^{k}$ for a specific feature $j$ employs $f_{j, t-1}^{k}$ in the calculation of $\hat{y}$. For the factors of all other features, the newest available values are used, i.e., depending on the sequence of features in the algorithm, either from the current $(\tau=t)$ or the preceding iteration $(\tau=t-1)$.

4. Quit when stopping criteria are met at the end of a full feature cycle.

This iterative cyclic optimization corresponds to a coordinate descent algorithm [17] with a boosting-like update of the factors $f$ and intrinsically supports the modeling of hierarchical causal dependencies in the data by means of choosing an appropriate feature sequence.

In order to increase robustness of the optimization and, if desired, reduce dependency on the sequence of features, a learning rate $\eta$ can be added to the calculation of the factors $f$ in eqn. 2 (with $\ln$ as link function):

$$
\ln \left(\tilde{g}_{j, t}^{k}\right)=\eta_{t} \cdot \ln \left(g_{j, t}^{k}\right) \text { where } \eta_{t} \in(0,1]
$$

Here, $\eta$ is chosen as a small value at the beginning of the training $(t=1)$ and is then increased after each full feature cycle $t$ according to a linear or logistic function until it reaches $\eta=1$ for the maximal number of iterations, hence $\tilde{g}_{j}^{k} \rightarrow g_{j}^{k}$ as the algorithm converges.

If the values $y$ follow a Poisson distribution, the Cyclic Boosting algorithm corresponds to optimizing $\sum_{i} \frac{\left(y_{i} / \hat{y}_{i, \tau}-g_{j}^{k}\right)^{2}}{\sigma_{i}^{2}}$, i.e. $\chi^{2}$, with $\sigma_{i}^{2}=y_{i} / \hat{y}_{i, \tau}$ for all observations $i$ in each bin $k$ of feature $j$. Since the bins of each feature variable are considered independently of each other, the optimization is performed locally in each bin $b_{j}^{k}$. This has the benefit that rare events can be learned effectively by the algorithm. While most machine learning algorithms tend to over-regularize these effects, especially when they are far away from the bulk of the respective distribution of observed feature variables $X_{j}$, choosing a suitable binning allows to treat rare observations separately from the bulk of the distribution of observed feature variables and hence allow accurate predictions even in this case. However, the potentially low numbers of observations in such bins increase the need for regularization methods in order to avoid learning wrong or spurious relationships from data, i.e. reduce the risk of overfitting.
Although the cyclic consideration of all variables already accounts for correlations between the different features, the learning of correlations between specific features can be further improved by adding composed features with multi-dimensional binning, e.g. built out of two or three of the original features. An example for this is described later on in figure 2 .

The binned feature-wise optimization of the Cyclic Boosting method also enables a natural access to the introduction of sample weights. As an example, this can be used to put more emphasis on the most recent past when predicting a target available as times series data. Such a procedure can help to improve the forecast quality in case of trends or other temporal changes in the data.

Owing to its straightforward structure based on fundamental arithmetic operations, Cyclic Boosting can be trained efficiently on a large amount of data and parallelization of the algorithm is possible without major obstacles.

\subsection{Regularization}

The factors $f_{j}^{k}$ are iteratively updated according to eqn. 2 . where the update rule has the form $g=\alpha / \beta$. As the Gamma distribution is the maximum entropy probability distribution for a random variable $\xi$ for which $E[\xi]=\alpha / \beta$ is fixed and greater than zero, the Gamma distribution is assumed as a prior for the distribution of the factors $f_{j}^{k}$ in each bin $k$ of feature $j$. Furthermore, the numerator and denominator of eqn. 2 have the form of the maximum likelihood estimator for an i.i.d. random variable following a Poisson (or more general negative binomial) distribution. These considerations motivate the description of the individual contributions, i.e. the factors, to the prediction of a target variable $Y \in[0, \infty)$ as conjugate distributions, the Gamma distribution being the conjugate prior to the Poisson (or more general negative binomial) likelihood. Eqn. 2 can hence be written as:

$$
g_{j}^{k}=\frac{\alpha_{j}^{k}}{\beta_{j}^{k}}
$$

with

$$
\alpha_{j}^{k}=\alpha_{\text {prior }}+\sum_{x_{j, i} \in b_{j}^{k}} y_{i} \text { and } \beta_{j}^{k}=\beta_{\text {prior }}+\sum_{x_{j, i} \in b_{j}^{k}} \hat{y}_{i}
$$

The numerical values of the parameters of the prior Gamma distribution are chosen such that the median of the Gamma distribution is 1 , i.e. $\alpha_{\text {prior }}=2, \beta_{\text {prior }}=1.67834$.

The definition of the factors in eqn. 5 exploits the fact that the mean of the Gamma distribution can be expressed as $\alpha / \beta$. Instead, one could also choose the median, which is generally a more robust point estimator and not as sensitive to outliers as the mean.

\subsection{Smoothing}

In most realistic applications, the observed data will be noisy and subject to statistical fluctuations, assuming that missing, incomplete or wrong data have already been corrected and common best practices for improving data quality have been observed. Regularizing the factors $f_{j}^{k}$ 
across bins $k$ for each feature $j$ will therefore improve the numerical stability of the algorithm training. For categorical features, the factors in each category can be regularized by determining appropriate Bayesian a priori probabilities for each occurrence of the specific category of feature variable $X_{j}$. For continuous features, smoothing functions such as splines or a suitable base of orthogonal polynomials can be applied, which is equivalent to applying a low-pass filter to remove high-frequency noise.

It should be noted that the range of the factors need to be transformed from $(0, \infty)$ to $(-\infty, \infty)$ before these smoothing approaches can be applied. This can be achieved by taking the logarithm of the factors, i.e. $f_{j}^{\prime k}=\ln \left(f_{j}^{k}\right)$. In order to be able to fit a smoothing function to the factors, the uncertainties $\sigma_{f_{j}^{\prime k}}$ of each factor $f^{\prime}$ in each bin $k$ for feature $j$ can be estimated from moment matching of the Gamma distribution to the log-normal distribution, i.e. assuming that the uncertainties follow a Gaussian distribution after the logarithmic transformation has been applied. This means the variance of the Gamma distribution is set equal to the variance of the log-normal distribution:

$$
\frac{\alpha}{\beta^{2}}=\left(e^{\sigma^{2}}-1\right) \cdot e^{2\left(\mu+\frac{\sigma^{2}}{2}\right)}
$$

The mean of the log-normal distribution is then substituted by the mean of the Gamma distribution: $e^{\mu+\frac{\sigma^{2}}{2}}=\alpha / \beta$. And finally, this leads to the following formula for the uncertainties:

$$
\sigma_{f_{j}^{\prime k}}^{2}=\log \left(1+\alpha_{j}^{k}\right)-\log \left(\alpha_{j}^{k}\right)
$$

After the smoothing of the factors has been performed, the factors are transformed back to the original range (i.e. $(-\infty, \infty) \rightarrow(0, \infty))$ by applying the exponential function as the inverse of the natural logarithm.

\subsection{Additive regression mode}

In the additive regression mode, with the range of the target variable being $Y \in(-\infty, \infty)$, the formulae are modified such that:

$$
\begin{gathered}
\hat{y}_{i}=\mu+\sum_{j=1}^{p} f_{j}^{k} \quad \text { with } k=\left\{x_{j, i} \in b_{j}^{k}\right\} \\
f_{j, t}^{k}=\sum_{s=1}^{t} g_{j, s}^{k} \text { and } g_{j, t}^{k}=\sum_{x_{j, i} \in b_{j}^{k}} y_{i}-\sum_{x_{j, i} \in b_{j}^{k}} \hat{y}_{i, \tau}
\end{gathered}
$$

The conjugate distributions for the individual contributions to the prediction, in this case the summands, follow a Gaussian function. Therefore, no transformation is needed before smoothing.

\subsection{Classification mode}

In the case of (binary) classification, one aims to identify whether a given observation $i$ belongs to a certain class or not. Hence the range of the target variable is in $[0,1]$, which can be interpreted as the probability $p_{i}$ that this observation belongs to the class $\left(p_{i} \rightarrow 1\right)$ or doesn't belong to the class $\left(p_{i} \rightarrow 0\right)$. In practical applications, a suitable cut-off has to be defined which separates the two cases.

Noting that the odds, i.e. the ratio $\frac{p_{i}}{1-p_{i}}$, has the range $[0, \infty)$, the same approach as the multiplicative regression mode can be used:

$$
\frac{\hat{p}_{i}}{1-\hat{p}_{i}}=\mu \cdot \prod_{j=1}^{p} f_{j}^{k} \quad \text { with } k=\left\{x_{j, i} \in b_{j}^{k}\right\}
$$

Instead of a Gamma function, the conjugate prior for the factors is now a Beta function, due to the binary nature of the setting, and the corresponding likelihood is a Bernoulli distribution. Choosing $\alpha_{\text {prior }}=1.001$ and $\beta_{\text {prior }}=1.001$ results in a uniform Beta distribution for the prior that drops sharply to zero at either end of the interval $[0,1]$, which is helpful to avoid overconfidence with extreme predictions. The parameters of the posterior Beta distribution are then calculated as:

$$
\alpha_{j}^{k}=\alpha_{\text {prior }}+\sum_{x_{j, i} \in b_{j}^{k}} y_{i} \text { and } \beta_{j}^{k}=\beta_{\text {prior }}+\sum_{x_{j, i} \in b_{j}^{k}} 1-y_{i}
$$

The factors and their uncertainties are in turn estimated from the mean (or median) and variance of this Beta distribution, similar to the approach taken for the multiplicative regression mode.

The performance of the algorithm can be improved by the inclusion of sample weights according to the following scheme:

$$
w_{i}= \begin{cases}1-\hat{p}_{i}, & \text { if } y_{i}=1 \\ \hat{p}_{i}, & \text { if } y_{i}=0\end{cases}
$$

Similar to the approach taken in boosting, i.e. the combination of several weak learners into a strong one, this definition enforces the training process to put more emphasis on observations that have been misclassified in the current state of the algorithm. Eqn. 12 then reads:

$$
\begin{array}{r}
\alpha_{j}^{k}=\alpha_{\text {prior }}+\frac{\sum_{x_{j, i} \in b_{j}^{k}} w_{i} \cdot y_{i}}{\sum_{x_{j, i} \in b_{j}^{k}} w_{i}} \\
\beta_{j}^{k}=\beta_{\text {prior }}+\frac{\sum_{x_{j, i} \in b_{j}^{k}} w_{i} \cdot\left(1-y_{i}\right)}{\sum_{x_{j, i} \in b_{j}^{k}} w_{i}}
\end{array}
$$

Like in the multiplicative regression mode, the logarithm is then used to transform the range $(0, \infty)$ to $(-\infty, \infty)$ and in turn the same approach to regularization and smoothing can be taken.

\section{Example: Demand Forecasting}

A very useful application of Cyclic Boosting's multiplicative regression mode is to forecast future demand of individual products sold in a retail location. Hereby, demand is influenced by promotions, price changes, rebates, coupons, and even cannibalization effects within the assortment 
range. Furthermore, customer behavior is not uniform but varies throughout the week and is influenced by seasonal effects and the local weather, as well as many other contributing factors. Hence, even though demand generally follows a negative binomial distribution [18, the exact values of the parameters are specific to a single product to be sold on a specific day in a specific location or sales channel and depend on the wide range of frequently changing influencing factors mentioned above.

Cyclic Boosting allows to efficiently calculate all relevant parameters to model the demand of individual products, taking a wide range of influencing factors into account, while at the same time allowing the operational business to track and understand how each individual prediction was made.

\subsection{Data and algorithm training}

We use data from a Kaggle online competition [19 to demonstrate demand forecasting with Cyclic Boosting and showcase its properties. The data set consists of the fields date, store, item, and sales, the latter being the target to predict. There are five years of historical data, from beginning of 2013 until end of 2017, for 10 different stores and 50 different items.

Besides store and item, we include several features describing trend and seasonality, namely days since beginning of 2013 as linear trend as well as day of week, day of year, month, and week of month. A list of all used features (oneand two-dimensional) can be found in the legend of fig. 3. For example, two-dimensional features including the variable "item" allow to learn characteristics of time series of individual products.

Exemplarily, fig. 1 shows a detailed analysis of the factors for the feature variable "item". Shown are the mean values of the prediction $\hat{y}$ after completion of the training as well as the observed, true values $y$ in each bin divided by the global mean. This visualization directly indicates possible deviations from the optimal fit results in the different bins. Here, no significant deviations are present across the whole range of values. Furthermore, the smoothed values of the factors, i.e. the actual fitted parameters of the model, are shown. These differ from the mean values of the target and prediction in the different bins divided by the global mean due to correlations with other features.

An example for a two-dimensional feature combination, namely "store" and trend "td", is shown in fig. 2. The upper left-hand plot shows a binned, two-dimensional, color-coded visualization of the deviations between final predictions and truth. The lower left-hand plot shows the smoothed values of the two-dimensional factors, again visualized by means of color-coding. Here, one of the features is categorical ("store") and the other one continuous ("td"), and the two-dimensional smoothing is performed by means of grouping by the categorical feature dimension and smoothing the continuous one. An alternative for a two-dimensional smoothing in case of two continuous features consists in performing a truncated singular-value decomposition. The two right-hand plots show the two corresponding marginal smoothed factor distributions for

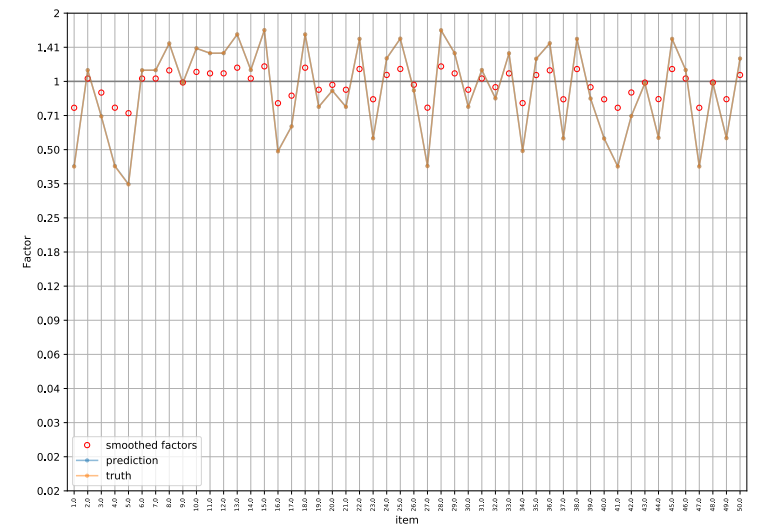

Figure 1: Analysis of the feature variable "item" after the final iteration. The shown data points indicate mean values of prediction $\hat{y}$ and ground truth $y$ for each bin divided by the global mean (prediction hardly visible due to good agreement). Note that the smoothed factors shown here are influenced by correlations to all other features in the model as well.

the mean of the respective other dimension (solid red) and its individual categories (transparent blue) as well as the marginal distributions for final predictions and observed (true) values.

These examples show how Cyclic Boosting supports model development in terms of feature engineering by means of analysis and individual preprocessing.

\subsection{Explanation of the predictions}

As stated above, one of the advantages of the Cyclic Boosting algorithm is that each individual prediction can be interpreted and related to the feature variables used as input, as shown in fig. 3 for three different predictions $\hat{y}_{i}$. A value of $f_{j}^{k}=1$ implies that the importance of this particular feature is neutral compared to the others, the strength of the deviation $f_{j}^{k} \neq 1$ indicates how important a given feature is for the individual prediction. As the figure illustrates, the importance of the individual features, from which the final prediction is calculated, can vary significantly from one observation to the next.

\subsection{Results}

The published results of the competition correspond to a test period from beginning of January to end of March 2018 [19]. However, the true observed values in this test period are not publicly available. Since the main aim of this example is to demonstrate that the Cyclic Boosting algorithm achieves at least comparable performance to other machine learning approaches while retaining the benefit of fully explainable predictions, the data until the end of 2016 were used for training the model and the first three months of 2017 were taken as an independent test 

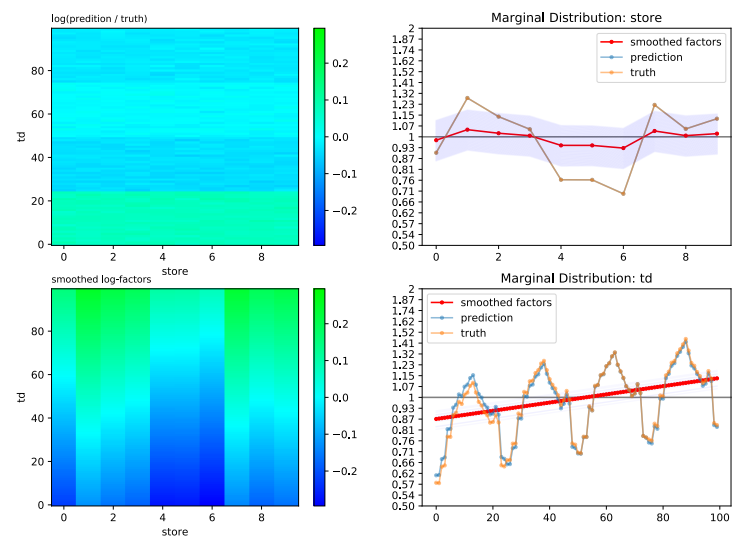

Figure 2: Analysis of the two-dimensional combination of the features "store" and "td" after the final iteration. The upper left-hand plot shows the two-dimensional deviations between prediction $\hat{y}$ and ground truth $y$, the lower left-hand plot the smoothed factors, and the right-hand plots $\hat{y}$ and $y$ for the two marginal distributions.

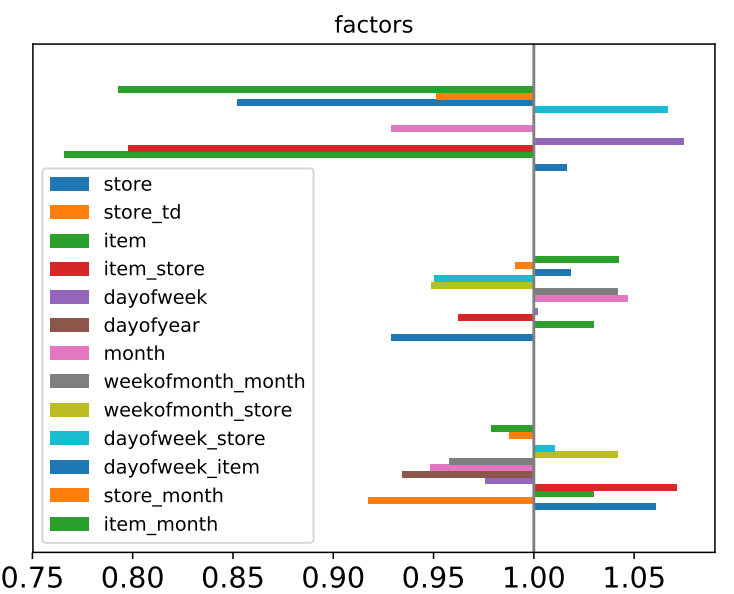

Figure 3: Illustration of the individual factors $f_{j}^{k}$ from which the prediction $\hat{y}_{i}$ is calculated for three individual observations (displayed on top of each other). sample. This reproduces the conditions of the competition as closely as possible and allows for a like-for-like comparison of the published results and scores with the results obtained from this model. Using the observed sales in the first three months of 2017 and comparing these to the predicted values, the approach using Cyclic Boosting results in a symmetric mean absolute percentage error of $\mathrm{SMAPE} \approx 13.20 \%$. The same approach with a training period until end of 2015 and prediction of the first three months of 2016 yields SMAPE $\approx 13.57 \%$. The winning models of the competition have scores of SMAPE $\approx 13.84 \%$ and SMAPE $\approx 12.58 \%$ for $34 \%$ and $66 \%$ of the data set for the first three months in 2018. Given the upward trend of sales and the larger training data set, we expect the SMAPE of our model in 2018 to be at least comparable to the winning method. One can therefore conclude that Cyclic Boosting can compete with other available algorithms in terms of forecast quality while retaining full explainability of the individual predictions.

In order to demonstrate the robustness of Cyclic Boosting against variations of the number of feature bins, which could be considered as hyperparameters, we both halved and doubled the number of bins (from default value of 100) for the two utilized continuous features, what resulted in changes in fourth place after the comma for SMAPE values.

As an additional remark, this simulated data set includes no information on prices, promotions, or product hierarchy and also shows no dependency on events, like holidays, weather, or other exogenous variables, for which the full potential of Cyclic Boosting would come to fruition.

\section{Discussion: Causality}

Causal dependencies play an instrumental role in any data generation process, and having reflected the underlying causal structure of the data, at least partially, in a supervised machine learning model is therefore beneficial for the generalization of the model and crucial for the interpretability of the learned correlations in terms of causal what-if scenarios for potential interventions [20,21].

\subsection{Individual causal effects}

The estimation of individual causal effects, suffering from the problem of counterfactuals, needs a method capable of generalizations by means of sample attributes, which is one of the key strengths of machine learning. In the sense of potential outcomes [22, a previously trained supervised machine learning model can be used to predict what-if scenarios for different values of one of its features.

Instead of the detour via two separate predictions, a direct prediction of absolute individual causal effects can be achieved with a machine learning algorithm capable of processing negative sample weights by subtracting one of the two potential outcome or what-if groups statistically from the other in the training. We call this technique statistical background subtraction. Due to its intrinsic feature binning, Cyclic Boosting in its additive regression mode (with adapted summand uncertainties) is ideally suited for this purpose: The external weights reflecting 
the respective membership to the two what-if groups $(+1$ and -1 in the case of certain memberships, positive and negative real values in the case of statistically estimated memberships 23 25]), are simply applied to the sums over all samples in each feature bin in eqn. 2, corresponding to the filling of the feature summand histograms. The accordingly weighted global average $\mu$ in eqn. 9 then corresponds to the average causal effect.

As an example, we look at the prediction of the individual causal effects of personalized coupons on customer demand (in terms of revenue) from the perspective of the retailer, and consider the simple case where unconfounded data from past random coupon assignments are available for the model training (see sec. 5.2 for a discussion of confounding). Each training sample then represents an individual customer, the values +1 and -1 are used as sample weights for customers that did or did not receive a coupon, respectively, and the target is the corresponding revenue from that customer in some defined time period, e.g., a week. The resulting predictions of this model then correspond to the absolute individual causal effects of coupon sending on revenue. Note that this effect can be both positive or negative, an example for the latter being customers that would have bought also without coupon for a higher price.

Another benefit of the proposed combination of machine learning with statistical background subtraction, namely the focus of the model training on the causal effect to be learned, can be seen in our couponing example when considering that most of the customers just ignore a coupon completely, for instance by not showing any demand in the time period at hand, no matter if they received a coupon for it or not. Due to the random coupon sending, these customers are present to the same extent in both groups (with weights +1 and -1 , respectively) of the data set used for the training, and are thus, thanks to the statistical background subtraction, effectively ignored in the model. In turn, the model can focus on learning the causal effect of the intervention on the target, rather than mainly learning the general dependencies of the target.

\subsection{Confounding}

There is one issue though with this approach of predicting individual causal effects with machine learning: Since the dependencies exploited by machine learning methods are merely statistical, the underlying causal structures of the data are not necessarily reflected in the learned model, because confounding effects can lead to spurious correlations overlaying the causal dependencies both between the different features and between these and the target.

The safest and most direct way to get rid of any confounding affecting an examined causal effect is via random assignment of the variable representing the cause, also known as randomized controlled trials 26. In case random assignment is not possible in a study or you are left with pure observational rather than interventional data, there is the need for a statistical method to avoid confounding of the data used for the training of the machine learning model, one example being independence weighting by means of inverse propensity scores 27]. For this, each input sample for the training is weighted by the inverse of the corresponding propensity score value, which can, for example, be calculated by a separate machine learning model trained on the observational values of the variable representing the cause and containing all potential confounders as features.

Rather than eliminating confounding in the data, Cyclic Boosting also allows to impose causal assumptions during the model training process itself, either via exploiting the order of features, as mentioned in sec. 3.2, or by utilizing feature-specific smoothing functions over the factors for the different bins, in order to restrict the learning of the dependency between the target and potentially confounding or confounded features to defined parametric forms, e.g., monotonous functions. For potential confounders, the idea is to enforce the model to describe specific causal effects by other features, namely the true causes. For example, yearly seasonality in a demand forecasting model could be smoothed by a sinusoidal curve, leaving the description of distinct peaking structures to other features like holidays or promotions. Group-by smoothing can be used for two-dimensional features consisting of a cofounder and an interventional variable to stratify the confounders. The restriction of interventional features to specific functional forms, for example an exponential price-demand elasticity in retail demand forecasting, can also help to extrapolate beyond the range of the observations in the training data.

Besides the use case of causal inference described in sec. 5.1, such incorporation of causal assumptions into a supervised machine learning model is also beneficial in terms of general forecasting quality, because it can improve the generalizability of the learned model. For a discussion of temporal confounding in time series forecasting see 28 .

\section{Conclusion}

A new machine learning algorithm, called Cyclic Boosting, was presented, which can be categorized as a generalized additive model with a cyclic coordinate descent optimization featuring a boosting-like update of parameters.

Cyclic Boosting addresses the challenge of prediction explainability on a fundamental level: Rather than relying on black box approaches, individual predictions $\hat{y}_{i}$ for single observations should not only be accurate but also explainable. Each prediction calculated using the Cyclic Boosting algorithm can be explained in terms of the strength of each feature variable contributing to the prediction.

Furthermore, Cyclic Boosting facilitates (multidimensional) feature engineering and enables the modeling of hierarchical causal dependencies and the prediction of rare effects in the data. Thereby, overfitting is effectively avoided by means of regularization and smoothing extensions.

Published at ICMLA 2019.

C)2020 IEEE. Personal use of this material is permitted. Permission from IEEE must be obtained for all other 
uses, in any current or future media, including reprinting/republishing this material for advertising or promotional purposes, creating new collective works, for resale or redistribution to servers or lists, or reuse of any copyrighted component of this work in other works.

Compared to the ICMLA paper (v2), we added (in v3) the discussion about causality in sec. 5 .

\section{References}

[1] M. Feindt and U. Kerzel, The NeuroBayes neural network package NIM A, vol. 559, no. 1, pp. 190-194, 2006 .

[2] T. Hastie and R. Tibshirani, Generalized Additive Models. Chapman and Hall/CRC, 1990.

[3] C. Molnar, Interpretable Machine Learning. 2019. https://christophm.github.io/ interpretable-ml-book/.

[4] L. S. Shapley, A Value for n-Person Games Cntributions to the Theory of Games II, Annals of Mathematics Studies, vol. 28, pp. 307-317, 1953.

[5] S. Lundberg and S. Lee, A unified approach to interpreting model predictions CoRR, vol. abs/1705.07874, 2017.

[6] L. Breiman, Random Forests Machine Learning, vol. 45, pp. 5-32, Oct 2001.

[7] TeamHG-Memex, "eli5: A library for debugging/inspecting machine learning classifiers and explaining their predictions." https://github.com/ TeamHG-Memex/eli5.

[8] Google, "The what-if tool: Code-free probing of machine learning models." https://github. com/tensorflow/tensorboard/tree/master/ tensorboard/plugins/interactive_inference.

[9] J. H. Friedman, Greedy function approximation: A gradient boosting machine. Ann. Statist., vol. 29, pp. 1189-1232, 102001.

[10] M. D. Zeiler and R. Fergus, Visualizing and Understanding Convolutional Networks arXiv preprint arXiv:1311.2901, 2013.

[11] K. Simonyan, A. Vedaldi, and A. Zisserman, Deep Inside Convolutional Networks: Visualising Image Classification Models and Saliency Maps arXiv preprint arXiv:1312.6034, 2013.

[12] M. T. Ribeiro, S. Singh, and C. Guestrin, "Why Should I Trust You?": Explaining the Predictions of Any Classifier in Proceedings of the 22nd ACM SIGKDD International Conference on Knowledge Discovery and Data Mining, San Francisco, CA, USA, August 13-17, 2016, pp. 1135-1144, 2016.

[13] J. A. Nelder and R. W. M. Wedderburn, Generalized Linear Models Journal of the Royal Statistical Society. Series A (General), vol. 135, no. 3, pp. 370-384, 1972.
[14] Microsoft, "Interpretml." microsoft/interpret.

[15] R. Caruana, Y. Lou, J. Gehrke, P. Koch, M. Sturm, and N. Elhadad, Intelligible Models for HealthCare: Predicting Pneumonia Risk and Hospital 30-day Readmission in Proceedings of the 21th ACM SIGKDD International Conference on Knowledge Discovery and Data Mining, KDD '15, (New York, NY, USA), pp. 1721-1730, ACM, 2015.

[16] B. E. Boser, I. M. Guyon, and V. N. Vapnik, A training algorithm for optimal margin classifiers in Proceedings of the fifth annual workshop on Computational learning theory - COLT '92, ACM Press, 1992.

[17] S. J. Wright, Coordinate descent algorithms Mathematical Programming, vol. 151, pp. 3-34, Jun 2015.

[18] A. S. C. Ehrenberg, The Pattern of Consumer Purchases Journal of the Royal Statistical Society. Series $C$ (Applied Statistics), vol. 8, no. 1, pp. 26-41, 1959.

[19] https://www.kaggle.com/c/ demand-forecasting-kernels-only/data.

[20] J. Pearl, Causality: Models, Reasoning and Inference. Cambridge University Press, 2 ed., 2009.

[21] J. Pearl, The Seven Tools of Causal Inference, with Reflections on Machine Learning Commun. ACM, vol. 62, pp. 54-60, Feb 2019.

[22] D. B. Rubin, Causal Inference Using Potential Outcomes Journal of the American Statistical Association, vol. 100, no. 469, pp. 322-331, 2005.

[23] M. Pivk and F. Le Diberder, : A statistical tool to unfold data distributions Nuclear Instruments and Methods in Physics Research Section A: Accelerators, Spectrometers, Detectors and Associated Equipment, vol. 555, p. 356?369, Dec 2005.

[24] CDF-Collaboration, Measurements of the properties of $\Lambda_{c}(2595), \Lambda_{c}(2625), \Sigma_{c}(2455)$, and $\Sigma_{c}(2520)$ baryons Phys. Rev. D, vol. 84, p. 012003, Jul 2011.

[25] CDF-Collaboration, Measurement of $C P$-violation asymmetries in $D^{0} \rightarrow K_{S}^{0} \pi^{+} \pi^{-}$Phys. Rev. D, vol. 86, p. 032007, Aug 2012.

[26] T. C. Chalmers, H. Smith, B. Blackburn, B. Silverman, B. Schroeder, D. Reitman, and A. Ambroz, A method for assessing the quality of a randomized control trial Controlled Clinical Trials, vol. 2, no. 1, pp. 31-49, 1981.

[27] P. R. Rosenbaum and D. B. Rubin, The central role of the propensity score in observational studies for causal effects Biometrika, vol. 70, pp. 41-55, 041983.

[28] F. Wick, U. Kerzel, M. Hahn, M. Wolf, T. Singhal, D. Stemmer, J. Ernst, and M. Feindt, Demand Forecasting of Individual Probability Density Functions with Machine Learning arXiv preprint arXiv:2009.07052v2, 2021. 